\title{
BULGE K AND M GIANTS
}

\author{
DONALD M. TERNDRUP \\ Department of Astronomy, The Ohio State University, \\ 174 W. 18th. Ave., Columbus, OH 49210 USA
}

\begin{abstract}
Several independent studies of the abundances and kinematics of $K$ and $M$ giants in the inner Galaxy $(R<2 \mathrm{kpc})$ are assembled to trace out this region's global properties. The mean metal abundance is $[\mathrm{M} / \mathrm{H}] \approx+0.3$ at $R=0.5 \mathrm{kpc}$, and declines by about 1.2 dex out to $R=2 \mathrm{kpc}$. The line-of-sight velocity dispersion at $R=0.5 \mathrm{kpc}$ is $\sigma_{r} \approx 115 \mathrm{~km} \mathrm{sec}^{-1}$ for all population tracers, and declines by $d \log \sigma_{r} / d \log R=-0.4$. It now seems fairly clear that only the most metal-poor $\mathrm{K}$ giants become RR Lyrae variables, while the more metal-rich ones become late $\mathrm{M}$ stars. There is some evidence that the most metal-rich stars are in a flatter, more rapidly rotating system. Metal abundance ratios for a few $\mathrm{K}$ giants suggest that the inner Galaxy may have formed rapidly.
\end{abstract}

Key words: Galactic Bulge - Late-type Stars - Metallicity Gradients - Stellar Abundances

\section{Introduction}

One of the recurrent themes of this meeting is that we do not have it clear in our heads what we mean when we use the word "bulge." Some of us use the word to describe the stellar population along lines of sight toward $|\ell|,|b|<10^{\circ}$ (approximately), and especially the very metal-rich $\mathrm{K}$ giants or highly evolved late-type stars found there. Others here refer to a component of the Galaxy that has a particular spatial distribution, boxy or roundish like the bulges of other galaxies, and perhaps containing bars or other non-axisymmetric features. The situation is further confused as we are try to distinguish the bulge from other components of the Galaxy, such as the disk or halo. Several of us here have repeatedly raised - without satisfactory answers - many questions about the whether disk, halo, and bulge stars are all mixed up in the inner Galaxy, or whether there is even any way of distinguishing these components with our current data.

I should like to argue, just for the duration of this talk, that we would be better off if we realized that the terms "bulge," "disk," and "halo" may have limited meaning in our studies of the inner Galaxy:

- $\quad$ Bulges and ill defined: We should remember that a given stellar population is defined either by its spatial distribution or kinematics or chemical abundance. The various structural or population meanings of bulge, disk, and halo which we are using here are not equivalent to one another, especially since the defining populations for these groups are located in very different locations in the Galaxy.

- $\quad$ The bulge is complex: Any stellar population can be thought of as a distribution of points in a parameter space with axes of age, chemical abundances, kinematics, etc. The case can be made (e.g., Carney et al. 1990) that in the solar neighborhood, the disk and halo have nearly disjoint distributions in (say) $[\mathrm{Fe} / \mathrm{H}]$ and kinematics, though intermediate populations, such as the thick disk, have properties that overlap those of the disk and halo. This leads us to view the Galaxy as a superposition of well-defined populations, as Baade 
(1944) did when he defined his Populations I and II. Even if we have a complete map of the inner Galaxy in parameter space, however, we may not find that the Galaxy comes apart in that region into separable groups, some of which correspond to the disk or halo that we see locally. Indeed we know (e.g., Lewis and Freeman 1989) that the disk and bulge are kinematically inseparable within $R=1-2 \mathrm{kpc}$.

- Our data are incomplete: We still need to clarify the evolutionary relationship between the various tracers of the Galaxy's population and gravitational potential within $R=1 \mathrm{kpc}$. We are busy studying the spatial distribution, metallicities and kinematics of $K$ and M giants, RR Lyrae stars, LPVs, Masers, etc., which may have quite different ages or metallicities. Certain of these objects correspond to local halo stars (e.g., the metal poor ones) or to disk objects (e.g., those orbiting near the circular speed). We may, however, be examining parts of the inner Galaxy that are in different evolutionary stages, calling them all "bulge" objects, when in fact we are looking at portions of the inner Galaxy which are not related to one another in an evolutionary sense, though mixed spatially.

If we were to study the inner regions of the Galaxy or of an external system such as M 31 with a minimum of prejudices, we would probably go to the telescope and obtain long-slit spectra and surface photometry to derive the stellar spatial distribution, and radial dependence of metallicity, velocity dispersion, and projected rotation speed. We would then model the integrated light as the sum of a bulge and disk (or whatever other components we want); those who are in this business know that one can decompose a galaxy into bulge and disk in a variety of ways, usually reflecting somewhat the (pre)judgement of the observer. What $I$ intend with this review is to assemble the kinds of data for our own Galaxy that we would get for an external system, then see what these data tell us about the inner Galaxy as a whole.

\section{K Giants}

\subsection{Abundance indicators and Abundance patterns}

The metallicities of $\mathrm{K}$ giants in Baade's Window have been extensively studied because stars of any abundance become $\mathrm{K}$ giants in old populations; a well conducted survey should therefore yield the true abundance determination. Spectroscopic surveys by Whitford and Rich (1983) and Rich (1988) have shown that the mean metallicity in Baade's Window is very high $([\mathrm{Fe} / \mathrm{H}] \sim+0.3)$, and that there is a wide range of abundances present, from $[\mathrm{Fe} / \mathrm{H}]=-1.0$ to +0.7 or even higher. (The precise determination of the metallicity scale for such high-abundance stars is uncertain, but independently of the calibration about one third of the stars in Rich's sample are more strong-lined at a fixed color than anything in the solar neighborhood.) Tyson (1991 and this meeting) and Geisler and Friel (1992) have derived similar metallicity distributions using Washington photometry of the $\mathrm{K}$ giants in Baade's Window. 


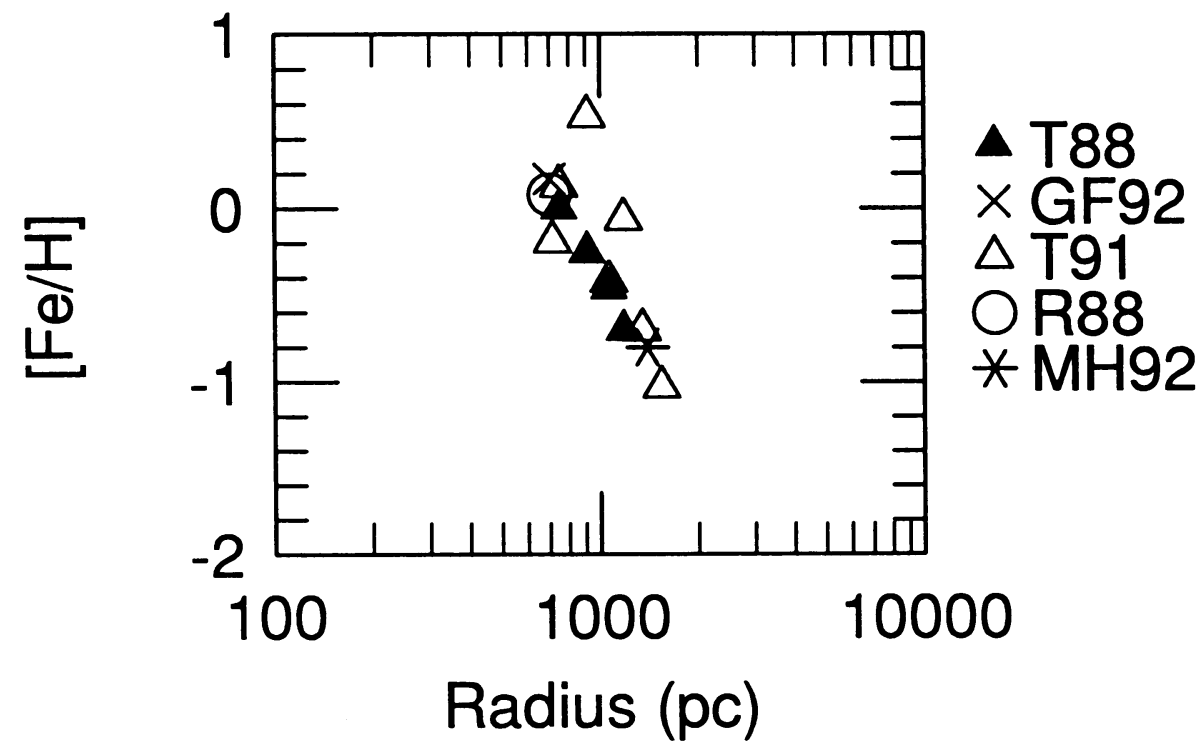

Fig. 1. Mean [Fe/H] of $\mathrm{K}$ giants in the inner Galaxy. References are: T88: Terndrup (1988); GF92: Geisler and Friel (1992), T91: Tyson (1991); R88: Rich (1988); MH92: Contributions by Harding and Morrison (this meeting).

In Figure 1 are plotted the mean $[\mathrm{Fe} / \mathrm{H}]$ of inner-Galaxy $\mathrm{K}$ giants as a function of galactocentric distance. The falloff in $[\mathrm{Fe} / \mathrm{H}]$ is about $1.2 \mathrm{dex}$ from $=0.3 \mathrm{kpc}$ to $R=2 \mathrm{kpc}$. Over this range, the surface brightness of the bulge falls off by about a factor of 100 (Terndrup 1988, Blanco 1988; see the discussion below). In each of these fields, the dispersion in metallicity is quite large, on the order of 0.75 dex.

Now all of the points on Figure 1 are from photometric studies or from lowresolution spectra, so should be considered as abundance indicators and not the true abundances one would derive from model atmosphere/line synthesis studies high-resolution spectra. Progress in obtaining true abundances in the inner Galaxy has been slow, since the relative faintness of bulge $\mathrm{K}$ giants $(V>16.5)$ has made high-resolution $\left(\lambda / \Delta \lambda>20 \times 10^{3}\right)$ studies of large samples very difficult. McWilliam and Rich (1992) have spectra of 12 Baade's Window K giants whose magnitudes and radial velocities give a high probability of membership in the inner Galaxy (rather than being nearby stars along the line of sight). They confirm the abundance scale found from the low-resolution spectra and find via analysis of equivalent widths that several of their stars exhibit a mild enrichment of the $\alpha$-elements, on the order of $[\alpha / \mathrm{Fe}]=+0.2$ to +0.3 . Perhaps most interesting is that they derive very high abundances of the r-process element Eu. As has been reviewed extensively (Tinsley 1980; Spite and Spite 1985; Gilmore et al. 1989; Wheeler et al. 1989), an enchancement of $\mathrm{Eu}$ or of the $\alpha$-capture elements indicates that the chemical 
enrichment was dominated by type II supernovae; these result from the explosion of single, massive $\left(30 M_{\odot}>M>10 M_{\odot}\right)$ stars that have main sequence lifetimes of $10^{9} \mathrm{yr}$ or less. The enrichment process in the bulge was therefore rapid, as in the halo. It is probably reasonable to expect that the bulge could have gone to very high metallicities in a short time: if the inner Galaxy began making stars with a high initial gas density, the evolutionary products would have been trapped in the bulge's deep gravitational potential, and so be available quickly for further star formation.

Peterson and Terndrup (1992) have obtained a high-resolution $(\lambda / \Delta \lambda=30 \times$ $\left.10^{3}\right)$ spectrum of one faint $(V=17.1)$ star in Baade's Window. Analysis of this spectrum was performed using model atmospheres and line synthesis as described by Peterson et al. (1992). The analysis is currently not complete, but preliminary results indicate that for this star at least, strong $\mathrm{CN}$ bands suggest an enchancement of nitrogen: $[\mathrm{N} / \mathrm{Fe}] \sim 0.4$ at $[\mathrm{Fe} / \mathrm{H}]=+0.45$. The star does not seem to have an observable oxygen enhancement, suggesting that the bulge may not be completely composed of stars formed in a rapid-enrichment scenario.

Now rapid formation does not necessarily imply that the bulge is extremely old, with the same age, for example, as the oldest globulars. In the discussions of the local halo, the statement is often made that the halo has to be old because halo stars have $[\alpha / \mathrm{Fe}]>0$ and the disk is younger because $[\alpha / \mathrm{Fe}] \sim 0$ (resulting from chemical evolution over time scales longer than $10^{9} \mathrm{yr}$ ). But strictly speaking the formation of the halo and disk could have been independent, as suggested by the nearly complete separation between the two components in metallicity and kinematics (e.g., Carney et al. 1990). The halo is old because its main-sequence stars are all of low mass; it formed quickly because the stars have $[\alpha / \mathrm{Fe}]>0$. Even if the inner-Galaxy stars all have $[\alpha / \mathrm{Fe}]>0$, we still have not measured the age.

The direct measures of the bulge turnoff in fields at $|b| \geq 8^{\circ}$ (Terndrup 1988) and in Baade's Window (Baum et al., this meeting), give a mean age of $\approx 10 \times 10^{9}$ yr. Though this value is rather uncertain, depending sensitively on adopted values for the bulge metallicity scale and line-of-sight extinction, it seems that the bulge is younger than the halo even though it, like the halo, may have formed rapidly ${ }^{1}$. This seems consistent with the views of Carney et al. (1990), who argued that the bulge and halo should be a natural evolutionary sequence, with the bulge formed from lost halo gas.

\section{M giants}

The bulge $\mathrm{M}$ giants have been the subject of many studies, since they are relatively bright in the red and infrared, and are easily detected in grism surveys (e.g., Blanco et al. 1984, Blanco and Terndrup 1989, and references in these papers). The metallicity scale for $\mathrm{M}$ giants is less certain than for $\mathrm{K}$ giants, since the atmospheres of such cool stars contain strong absorption from molecules and it is therefore hard to generate theoretical models for comparison to observed spectra. Bulge M giants

1 The mean age quoted by Terndrup (1988) - 11 to $14 \times 10^{9} \mathrm{yr}-$ was for an adopted distance of $R_{0}=7 \mathrm{kpc}$. For $R_{0}=8 \mathrm{kpc}$, the distance adopted by Baum et al., the mean age would be 9 to $12 \times 10^{9} \mathrm{yr}$; the two studies are therefore in agreement. 
have significantly stronger atomic and molecular absorption than do stars of the same temperature in the solar neighborhood or in globular clusters (Frogel and Whitford 1987; Frogel et al. 1990; Sharples et al. 1990; Terndrup et al. 1990, 1991); semi-quantitative analysis of the photometric and spectroscopic abundances of the Baade's Window $M$ giants in all these papers yield a mean metallicity of $[\mathrm{Fe} / \mathrm{H}]$ $\sim+0.2$ to +0.4 in Baade's Window, in agreement with mean metallicity of the $\mathrm{K}$ giants.

The $M$ giants show a decline in mean metallicity by a few tenths dex from $R=500 \mathrm{pc}$ to $R=1500 \mathrm{pc}$ (Terndrup 1988; Frogel et al. 1990; Terndrup et al. 1990; Tyson 1991). To illustrate this, I reproduce as Figure 2 the correlation of the strength of TiO absorption in bulge $\mathrm{M}$ giants as a function of infrared $J-K$ color for three groupings of stars along the minor axis of the bulge $\left(\ell=0^{\circ}\right)$; this plot is from Terndrup et al. (1990).

There is considerable evidence that the $\mathbf{M}$ giants, unlike the $\mathrm{K}$ giants, are a biased tracer of the bulge population, representing instead the metal-rich end of the metallicity distribution. The first piece of evidence, shown in Figure 3, is that the gradient in the area density of coolest $M$ giants is significantly steeper than that of the $\mathrm{K}$ giants or early $\mathrm{M}$ giants (Blanco 1988; Terndrup 1988). The bulge M2+ giants fall off with radius according to $\Sigma(R) \propto R^{-\nu}$, with $\nu=2.5$, like that of the integrated light (Kent 1992) or of the halo, but the falloff of $M$ giants of spectral type M7 or later is much steeper: more like $\nu=3.3$. The usual interpretation is that the giant branch shifts to warmer temperatures as the metallicity declines, so that fewer stars will have sufficiently cool temperatures to be late $\mathbf{M}$ giants.

The second piece of evidence is that there seems to be a lack of $M$ giants that have abundances like the most metal-poor $\mathrm{K}$ giants wherever both types of stars have been studied. In Figure 2, for example, the are practically no $\mathbf{M}$ giants in the $-3^{\circ}$ and -48 fields that have $\mathrm{TiO}$ strengths like solar-neighborhood stars, and none at all with strengths like 47 Tuc stars (i.e., weaker than those of the solarneighborhood stars at a given $J-K$ ), even though there are plenty of $\mathrm{K}$ giants in Baade's Window with this abundance. In fact several photometric and spectroscopic studies of bulge M giants (Frogel et al. 1990; Sharples et al. 1990; Terndrup et al. $1990,1991)$ all show that these late-type stars show a negligible dispersion in metal abundance; specifically they show that the dispersion in abundance indicators such as $\mathrm{CO}$ or $\mathrm{TiO}$ strength vs. $J-K$ is not larger than is produced by observational error alone. This would be true if only the most metal-rich third (say) of the $\mathrm{K}$ giants (which have a dispersion in metallicity of $\sim 0.7 \mathrm{dex}$ ) in the inner Galaxy were producing all the $M$ giants. The precision in the determination of metallicity in the current studies is about \pm 0.3 dex per star, which is about half the intrinsic dispersion of the $\mathrm{K}$ giants' metallicities, so all the $\mathrm{M}$ giants would look like they have the same abundance. Figure 4 shows a comparison of the abundance distribution for Baade's Window $M$ giants with that of the $\mathrm{K}$ giants; the $\mathrm{M}$ giant distribution is qualitatively shown as a gaussian of width 0.4 dex centered at $[\mathrm{Fe} / \mathrm{H}]=+0.3$.

Finally, Harding and Morrison (this meeting) show evidence that in a field at $R \sim 2 \mathrm{kpc}$, the kinematics of the $\mathrm{M}$ giants resembles that of the metal-rich $\mathrm{K}$ giants.

Given the situation in globular clusters, in which only the metal-rich ones produce $M$ giants, it is perhaps not surprising that only those stars on the metal-rich 


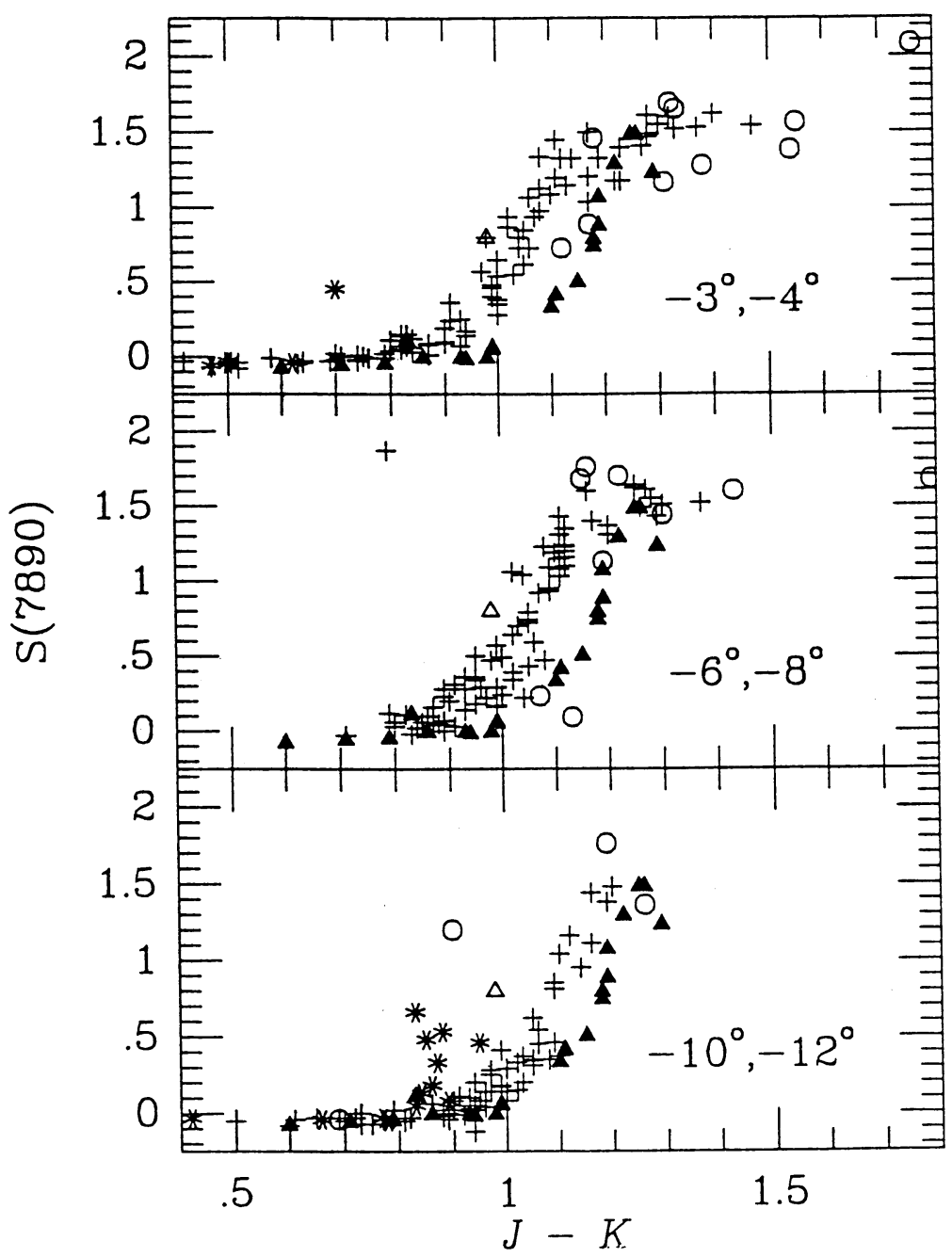

Fig. 2. Correlation between $\mathrm{S}(7890)$, a measure of the strength of $\mathrm{TiO}$ absorption near $7890 \AA$, and dereddened $J-K$ color. The data are for bulge fields along $\ell \approx 0^{\circ}$, grouped into three pairs of fields: for $b=-3^{\circ}$ and $-4^{\circ}$ (top panel), $b=-6^{\circ}$ and $-8^{\circ}$ (center), and $b=-10^{\circ}$ and $-12^{\circ}$ (lower panel). Symbols are: filled triangles, solar-neighborhood $\mathrm{M}$ stars; open triangle, the nearby $\mathrm{M}$ dwarf Wolf 359 ; plusses, bulge $\mathrm{M}$ giants that are not long-period variables; open circles, bulge long-period variables; and asterisks, foreground M dwarfs seen in front of the bulge. Data are from Terndrup et al. 1990. 


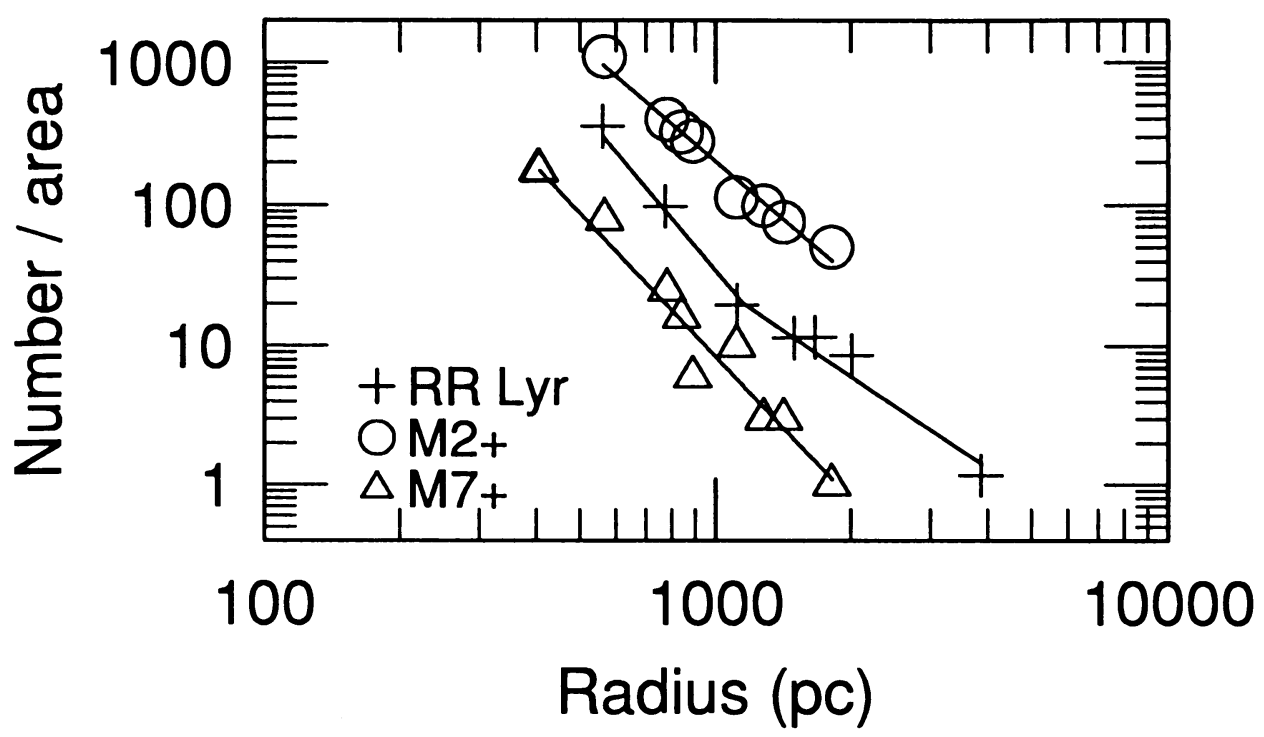

Fig. 3. Surface density of various tracers of the bulge population. The plusses are for RR Lyrae variables, the open circles are for M giants of type M2 or later, and the open triangles are for giants of type M7 or later.

tail of the bulge's $[\mathrm{Fe} / \mathrm{H}]$ distribution should produce $\mathrm{M}$ giants. But I find it odd nevertheless that there are few or no 47 Tuc-like $M$ giants in Baade's Window. If the bulge is somewhat younger than 47 Tuc, which is suggested by the color-magnitude diagrams of the turnoff, then the stars now going up the giant branch should be somewhat more massive, and even more likely to become $M$ giants than those in 47 Tuc. The only way they cannot become $M$ giants is if somehow these turnoff stars are less massive than those in 47 Tuc. Guided by the mass-age-metallicity relationship in VandenBerg and Laskarides (1987), the turnoff stars in that cluster should have $M=0.85 M_{\odot}$. Suppose this is the minimum mass necessary for an $M$ giant to be created, and we want to explain why the minimum metallicity of Baade's Window $\mathrm{M}$ giants is $[\mathrm{Fe} / \mathrm{H}] \sim 0$ (say); in other words we would like turnoff stars in Baade's Window to have $M=0.85 M_{\odot}$ at $[\mathrm{Fe} / \mathrm{H}]=0.0$. If the helium abundance $Y$ is 0.25 , the bulge stars at this metallicity would have an age of approximately $20 \times 10^{9} \mathrm{yr}$. On the other hand if the helium abundance were even a bit higher than 47 Tuc, say $Y=0.28$, then stars with $[\mathrm{Fe} / \mathrm{H}]=0$ and age $10 \times 10^{9}$ yr would have a mass of $0.9 M_{\odot}$. For $Y$ increasing by a few hundredths while $Z$ goes from 47 Tuc-like to solar implies $\Delta Y / \Delta Z=1-3$, not an unreasonable value. 


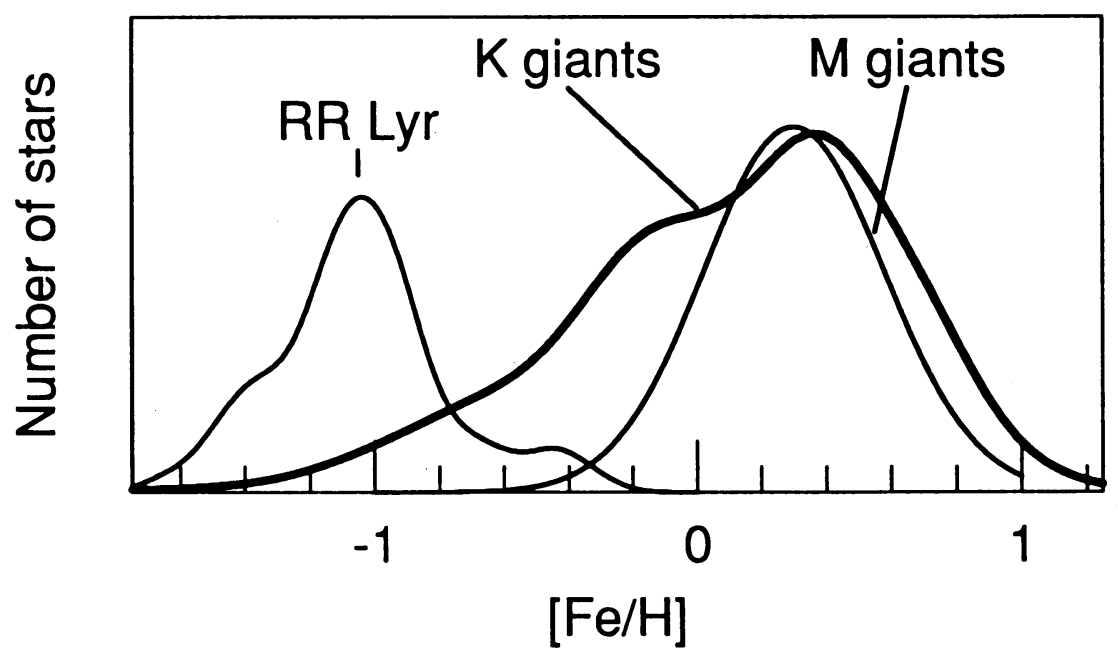

Fig. 4. Comparison of metallicity distributions in Baade's Window. The vertical scale and the relative numbers of stars under each distribution are arbitrary.

\section{The RR Lyraes and the $K$ giants}

The RR Lyraes in the bulge have a very different metallicity distribution than do the $\mathrm{K}$ giants. In Figure 4, I have plotted the RR Lyrae distribution from Walker and Terndrup (1991) along with the K giant distribution from Rich (1988); both distributions are shown as generalized histograms computed from the tabulated metallicities of individual stars. The RR Lyraes are evidently being produced from the most metal-poor tail of the $\mathrm{K}$ giants.

In Figure 3 are plotted the surface density of RR Lyrae variables from Blanco (1984) and from Oort and Plaut (1975). The kink in the solid line through the points for the RR Lyrae stars suggests that the surface density gradient may be steeper in the inner bulge than at larger galactocentric distances. (A single line fit through all the points has $d \log \Sigma / d \log R=-2.5$, like that for the M2+ giants.) Now if the bulge were behaving like a "first parameter" globular cluster, then we should expect that the surface density would be less steep than for the general population; as the metallicity increases toward the galactic center, the distribution of stars on the horizontal branch would shift to the cool side of the instability strip, and there should be proportionally fewer RR Lyrae variables. That the surface density steepens within $R=1 \mathrm{kpc}$ suggests that the inner bulge exhibits the "second parameter" effect.

Lee (1992) has recently analyzed the distribution of stars on the horizontal 
branches of globular clusters, in an attempt to see whether there is any variation with location in the Galaxy of the age spread among the globulars, assuming that age is the second parameter. Lee finds that globular clusters within $R=8 \mathrm{kpc}$ are all coeval, and on average about 2 Gyr older than those with $8 \leq R<40 \mathrm{kpc}$; clusters in this latter range of radius have an age spread of $3-\overline{4}$ Gyr. Such an interpretation is also suggested by Suntzeff et al. (1991) in their analysis of RR Lyraes in the field halo.

Lee also finds that the difference is mean metallicity between the RR Lyraes and the K giants in Baade's Window (see Figure 4) can only be explained if the bulge is $1-2 \times 10^{9}$ yr older than the halo globular clusters. The combination of old bulge and the age gradient with galactocentric radius leads Lee to propose that the bulge formed from the inside out. This is hard to reconcile with the observations from color-magnitude diagrams (Terndrup 1988; Baum et al., this meeting) that the bulge has a mean age near $10 \times 10^{9} \mathrm{yr}$, which suggests to me that there are many details of the evolution of bulge stars which remain to be understood.

\section{Kinematics}

\subsection{Bulge Kinematics}

To close, I would like to summarize the current information on bulge kinematics, and draw a few conclusions about the structure of the bulge and how stars of different metallicities may differ in spatial distribution. Rich (1990) and Sharples et al. (1990) have presented similar discussions in their studies of the $\mathrm{K}$ and $\mathrm{M}$ giants in Baade's Window.

The line-of-sight velocity dispersion in the inner Galaxy is a slow function of galactocentric radius. In Figure 5 are plotted values of $\sigma_{r}$ from several recent studies. These data are for $\mathrm{K}$ and $\mathrm{M}$ giants both along the minor axis of the bulge and for some fields several degrees away. The correlation between $\sigma_{r}$ and galactocentric distance $R$ is quite tight, with slope $d \log \sigma_{r} / d \log R=-0.4$. This behavior of $\sigma_{r}$ is also seen in Kent's (1992) kinematical model of the bulge.

In Figure 5 are shown two points of $\sigma_{r}$ from Rich (1990) and from Harding and Morrison (this meeting), who divide their sample into metal-poor and metal-rich stars; in each case, the metal-rich giants have a lower velocity dispersion, suggestive that they are confined to a flatter system with higher rotation speed Harding and Morrison show this explicitly in their off-axis field.

Possible correlations between metal-abundance and kinematics been explored extensively in Baade's Window. Data from this region are summarized in Table I. Rich (1990) presented velocities of $53 \mathrm{~K}$ giants in Baade's Window, and derived a line-of-sight radial velocity dispersion $\sigma_{r}=105 \pm 11 \mathrm{~km} \mathrm{sec}^{-1}$ for his full sample. He then divided his sample into bins of metallicity, and showed (though with small number statistics) that the radial velocity dispersion for the most metal rich stars $\left([\mathrm{Fe} / \mathrm{H} \geq+0.3)\right.$ was $92 \pm 14 \mathrm{~km} \mathrm{sec}^{-1}$, much lower than $126 \pm 22 \mathrm{~km} \mathrm{sec}^{-1}$ for stars with $[\mathrm{Fe} / \mathrm{H}]<-0.3$. This effect was also seen in the proper motion data of Spaenhauer et al. (1992), who measured relative proper motions in the $\ell$ and $b$ directions for over 400 stars in Baade's Window. (The proper motions are relative 


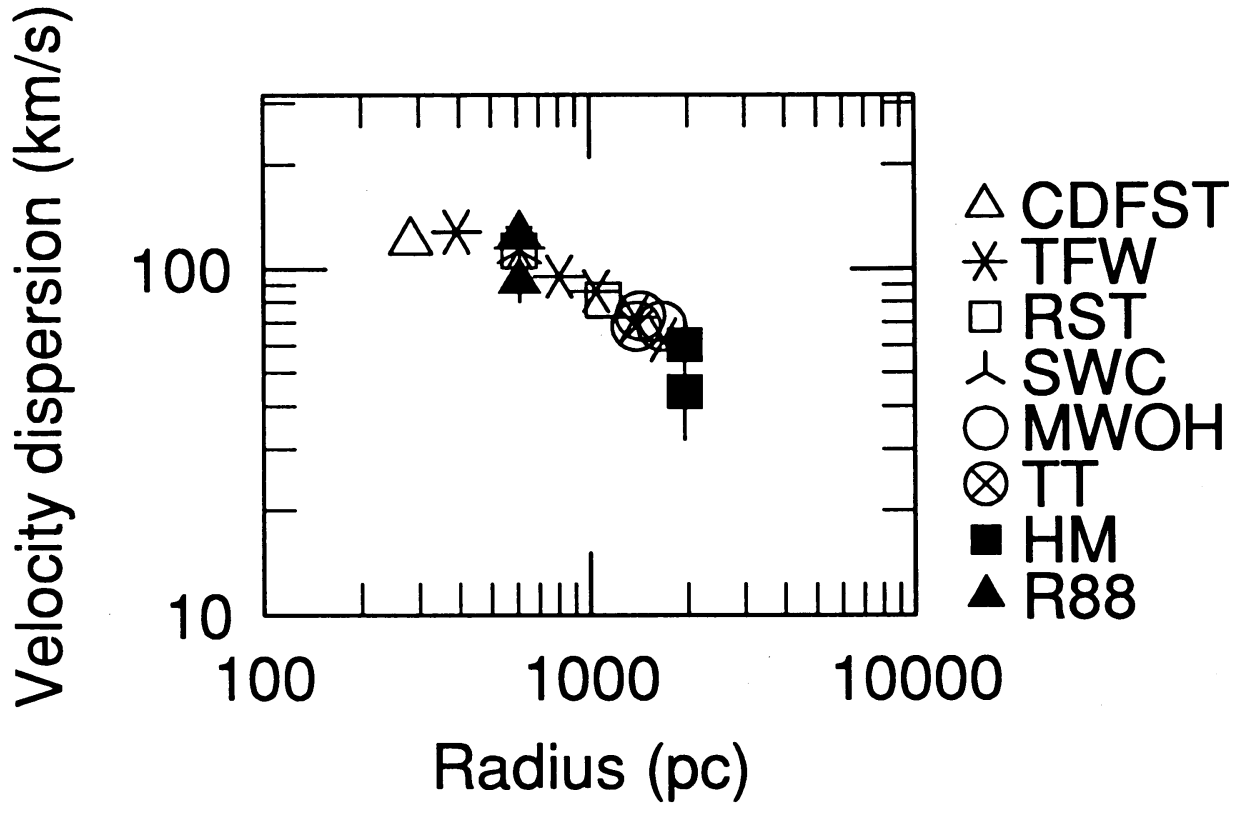

Fig. 5. Line-of-sight velocity dispersion in the bulge. The sources for the points are: CDFST: Carr et al. 1992; TFW: Terndrup et al. 1992; RST, Rich et al. 1992; SWC: Sharples et al. 1990, MWOH: Minniti et al. 1992; TT: Tiede and Terndrup 1992; HM: Harding and Morrison, this meeting; R88: Rich (1988).

TABLE I

Line-of-sight velocity dispersions in Baade's Window.

\begin{tabular}{lrcrr}
\hline Source & $\sigma_{r}$ & $\sigma_{\ell}$ & $\sigma_{b}$ & $\mathrm{~N}$ \\
\hline & & & & \\
Rich $(1990) \mathrm{K}$ giants & $105 \pm 11$ & $\ldots$ & $\ldots$ & 53 \\
Rich, [Fe/H] $<-0.3$ & $126 \pm 22$ & $\ldots$ & $\ldots$ & 16 \\
Rich, [Fe/H] $\geq+0.3$ & $92 \pm 14$ & $\ldots$ & $\ldots$ & 21 \\
Sharples et al. (1990) M5+ giants & $113 \pm 11$ & $\ldots$ & $\ldots$ & 225 \\
Spaenhauer et al. (1992) all stars & $\ldots$ & $115 \pm 4$ & $100 \pm 4$ & 429 \\
Spaenhauer et al. with [Fe/H] $\geq+0.0$ & $101 \pm 15$ & $118 \pm 14$ & $58 \pm 9$ & 34 \\
\hline
\end{tabular}


to the average of the sample because there are no background galaxies or proper motion standards visible in Baade's Window; their study therefore is a measure of the dispersion in the line-of-sight proper motion.) When they add radial velocities from Rich, Spaenhauer et al. find that the velocity dispersions in Baade's Window are nearly isotropic in projection, meaning the line-of-sight velocities are nearly equal: $\sigma_{r} \approx \sigma_{\ell} \approx \sigma_{b}$. When they use the small number of Rich's most metal-rich stars that are also in their sample, Spaenhauer et al. show that the vertical velocity dispersion $\sigma_{b} \sim 60 \mathrm{~km} \mathrm{sec}^{-1}$ is smaller than the tangential velocity dispersion $\sigma_{\ell}$.

That the projected velocity dispersion in Baade's Window is nearly isotropic may be a problem for some of the bar models for the bulge. A bar aimed somewhat towards us, as most of the current models indicate, ought to show $\sigma_{\ell}>\sigma_{r}$ and $\sigma_{\ell}>\sigma_{b}$. This does not seem to be the case, which suggests to me that the majority of Baade's Window stars cannot be in the bar. It may be the case that the expected bar signature is seen in the most metal-rich $\mathrm{K}$ giants in Baade's Window, but current statistics are not good enough to confirm this ${ }^{2}$. The only evidence that suggests that the metal-rich stars are in a bar is presented by Sharples et al. (1990), who have shown that the late-type $M$ giants in Baade's Window, which perhaps are the most metal-rich of the stars in that region, show evidence for a tangentially anisotropic velocity distribution.

The values of projected rotation speed have measured in several places in the inner Galaxy, as summarized in Figure 6.

Bulges of galaxies like our own (between Hubble type $\mathrm{Sb}$ and $\mathrm{Sc}$ ) are not selfgravitating bodies; the gravitational potential in the bulge has a very significant contribution from the disk. Consequently, the only proper way to explore kinematics in the bulge is to generate a mass model, as Kent (1992) has done from the Galaxy's $K$-band light distribution, and solve the equations relating kinematics and the density distribution. On the other hand, we can estimate how stars of different kinematics will be arranged in the bulge by making use of simple dynamical arguments, like those in Frenk and White's (1980) analysis of the globular cluster system. Suppose that stars near the mean of the bulge's metallicity distribution are arranged like $\Sigma \propto R^{-2.5}$, and are in a flattened axisymmetric bulge with axial ratio $c / a=0.7$ (Kent 1992). At the distance of the Baade's Window, these stars would mean rotation speed of $60-80 \mathrm{~km} \mathrm{sec}^{-1}$. If the metal-rich stars have $\sigma_{r}=90 \mathrm{~km}$ $\mathrm{sec}^{-1}$, as suggested by the data in Table 1 , then they would be in a significantly flatter system, one with $c / a \sim 0.4-0.5$ and have a projected surface density like $\Sigma \propto R^{-3.2}$, which is what the late $\mathrm{M}$ giants do.

So a first-order self-consistent picture the $M$ giants have the metallicities and kinematics that would arise if they are produced by the metal-rich tail of the $\mathrm{K}$ giant distribution. To confirm this, we would have to get much better abundances for both the $\mathrm{K}$ and $\mathrm{M}$ giants in several locations; a confirmation would also come with have well-understood star counts from which the evolutionary paths and rates can be derived. The big picture seems increasingly clear, but many details need to

\footnotetext{
2 I am part of a program which has obtained radial velocities and metallicities for the full Lick proper motion survey in Baade's Window; our results are now in preparation (Rich et al. 1992), and should indicate whether there really is a significant variation of kinematics with metallicity or whether there are kinematical substructures in the bulge
} 


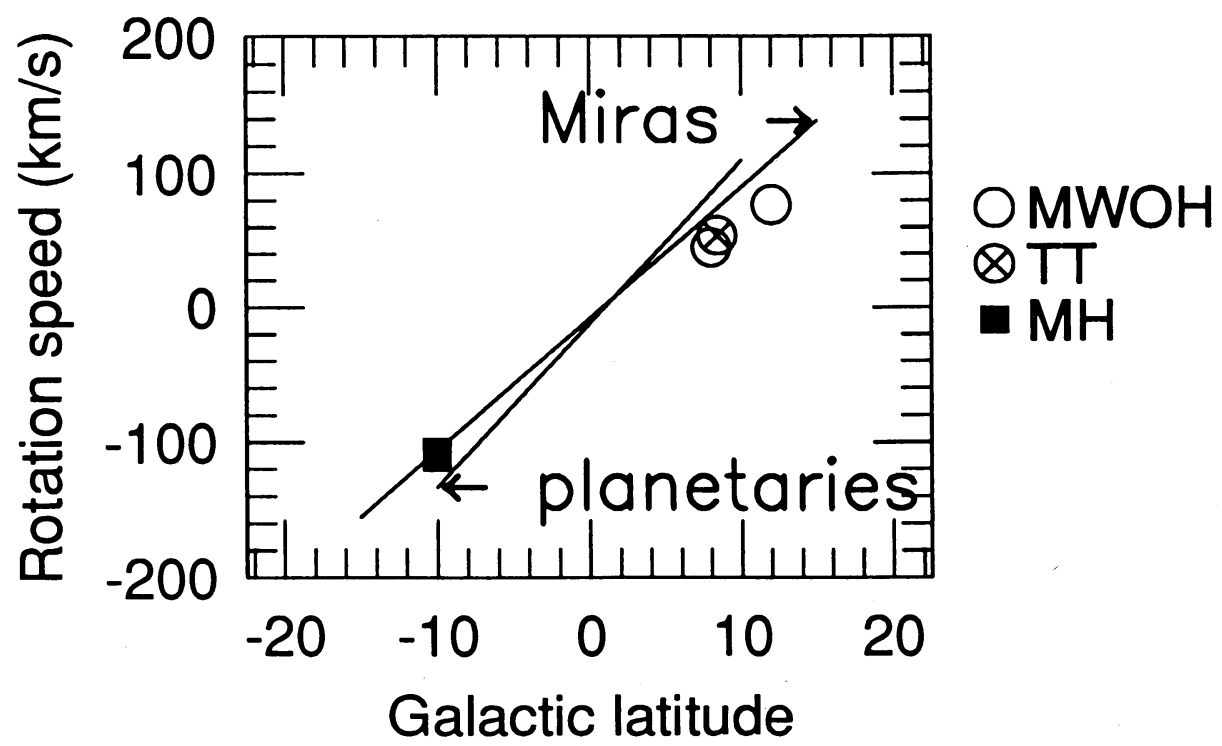

Fig. 6. Rotation velocity for various tracers in the inner Galaxy. The sources for the points are: MWOH: Minniti et al. 1992; TT: Tiede and Terndrup 1992; HM: Harding and Morrison, this meeting. The solid lines show the velocities found for Miras (Menzies 1990) and for bulge planetary nebulae (Kinman et al. 1988).

be filled in.

\section{References}

Baade, W. 1944, ApJ, 100, 137

Blanco, B. M. 1984, AJ, 89, 1836

Blanco, V. M. 1988, AJ, 95, 1400

Blanco, V. M., McCarthy, M. F., \& Blanco, B. M. 1984, AJ, 89, 636

Blanco, V. M., \& Terndrup, D. M. 1989, AJ, 98, 843

Carr, J., DePoy, D., Frogel, J. A., Sellgren, K., \& Terndrup, D. M. 1992, in preparation

Carney, B. W., Latham, D. W., \& Laird, J. B. 1990, AJ, 99, 572

Frenk, C. S., and White, S. D. M. 1980, MNRAS, 193, 295

Frogel, J. A., \& Whitford, A. E. 1987, ApJ, 320, 199

Frogel, J. A., Terndrup, D. M., Blanco, V. M., \& Whitford, A. E. 1990, ApJ, 353, 494

Geisler, D., \& Friel, E. D. 1992, AJ, 104, 128

Gilmore, G., Wyse, R. F. G., \& Kuijken, K., 1989, ARA\&A, 27, 555

Kent, S. M. 1992, ApJ, 387, 181

Kinman, T. D., Feast, M. W., \& Lasker, B. M. 1988, AJ, 95, 804

Lee, Y.-W. 1992, AJ, 104, 1780

Lewis, J. R., \& Freeman, K. C., 1989, AJ, 97, 139

McWilliam, A., \& Rich, R. M., 1992, in preparation. 
Menzies, J. W, 1990, in Bulges of Galaxies, eds. B. Jarvis \& D. Terndrup, ESO Conf. Ser., 35, 115

Minniti, D., White, S. D. M., Olszewski, E. W., \& Hill, J. M. 1992, ApJ, 393, L47

Oort, J., \& Plaut, L. 1975, A\&Ap, 41, 71

Peterson, R. C., \& Terndrup, D. M. 1992, in preparation

Peterson, R. C., Dalle Ore, C., \& Kurucz, R. 1992, ApJ, in press

Rich, R. M. 1988, AJ, 95, 828

Rich, R. M. 1990, ApJ, 362, 604

Rich, R. M., Sadler, E. M., \& Terndrup, D. M. 1992, in preparation

Sharples, R., Walker, A., \& Cropper, M. 1990, MNRAS, 246, 54

Spaenhauer, A., Jones, B. F., \& Whitford, A. E. 1992, AJ, 103, 297

Spite, M., \& Spite, F. 1985, ARA\&A, 23, 225

Suntzeff, N. B., Kinman, T. D., \& Kraft, R. P. 1991, ApJ, 367, 528

Terndrup, D.M 1988, ApJ, 96, 884

Terndrup, D. M., Frogel, J. A., \& Whitford, A. E. 1990, ApJ, 357, 453

Terndrup, D. M., Frogel, J. A., \& Whitford, A. E. 1991, ApJ, 378, 742

Terndrup, D. M., Frogel, J. A., \& Wells, L. A. 1992, in preparation

Tinsley, B M. 1980, Fund. Cosmic Phys., 5, 287

Tyson, N. D. 1991, Thesis, Princeton University

Walker, A. R., \& Terndrup, D. M. 1991, ApJ, 378, 119

Wheeler, J. C., Sneden, C., \& Truran, J. W. 1989, ARA\&A, 27, 279

Whitford, A. E. \& Rich, R. M. 1983, ApJ, 274, 723

VandenBerg, D. A., \& Laskarides, P. G. 1987, ApJS, 64, 103

\section{DISCUSSION}

Sellwood: In The Spaenhauer et.al. proper motion samples, for the $l$ and $b$ components, did you look to see if they were gaussian?

Tendrup: Sadler has done this for the whole sample. If you look at the $(l, b)$ plot, it forms a sort of round web. It isn't strongly tilted one way or the other.

Rich: I find it interesting that the M-giants seem to change in abundance as one goes from the inner to the outer fields. Yet, all the populations seem to share a single fall of velocity dispersion along the minor axis. This includes RR-Lyraes that were found by Rogers (1977), that seemed at a very low velocity dispersion, about $60 \mathrm{~km} / \mathrm{s}$ at -10 degrees.

Tendrup: I agree. The differences in velocity dispersion between stars of 2 dex in metallicity are subtle. They were $1 \sigma$ in your work and $1.5 \sigma-2 \sigma$ now, even in our new survey of over 400 stars. You must have very large samples in individual lines of sight to be able to make good statistical claims. I think that simply what is going on is that the gravitational potential has a certain shape and within that potential there were small changes in the kinematics as the chemical enrichment proceeded. 
King: If they have the same velocity dispersion in the centre and if they have anything like a gaussian velocity distribution, then they must have the same run of velocity dispersion with distance from the centre. Gravitation doesn't know about abundances, they're just test particles.

Norman: Is your metal rich population, of flattened stars, old or young?

Tendrup: It hasn't been looked into and I think it would be extraordinarily difficult to do, because you would need to know the details of determining the ages for individual stars. This means building really trustworthy stellar atmospheres models that would let you point to the spectrum and say "I know the gravity of this star" and that's tough! You could do statistical analysis, for example, on the main sequence and ask why the turnoff is so fat. Is it consistent with a rapid formation or with a big spread of abundances etc...

Morrison: I think that RR-Lyrae kinematics may give more information about age, this should be looked into.

Rich: We also need more complete samples of Miras, especially in identifying the longest period Miras. This may give us more direct information about the period and kinematics and any possible correlation between them.

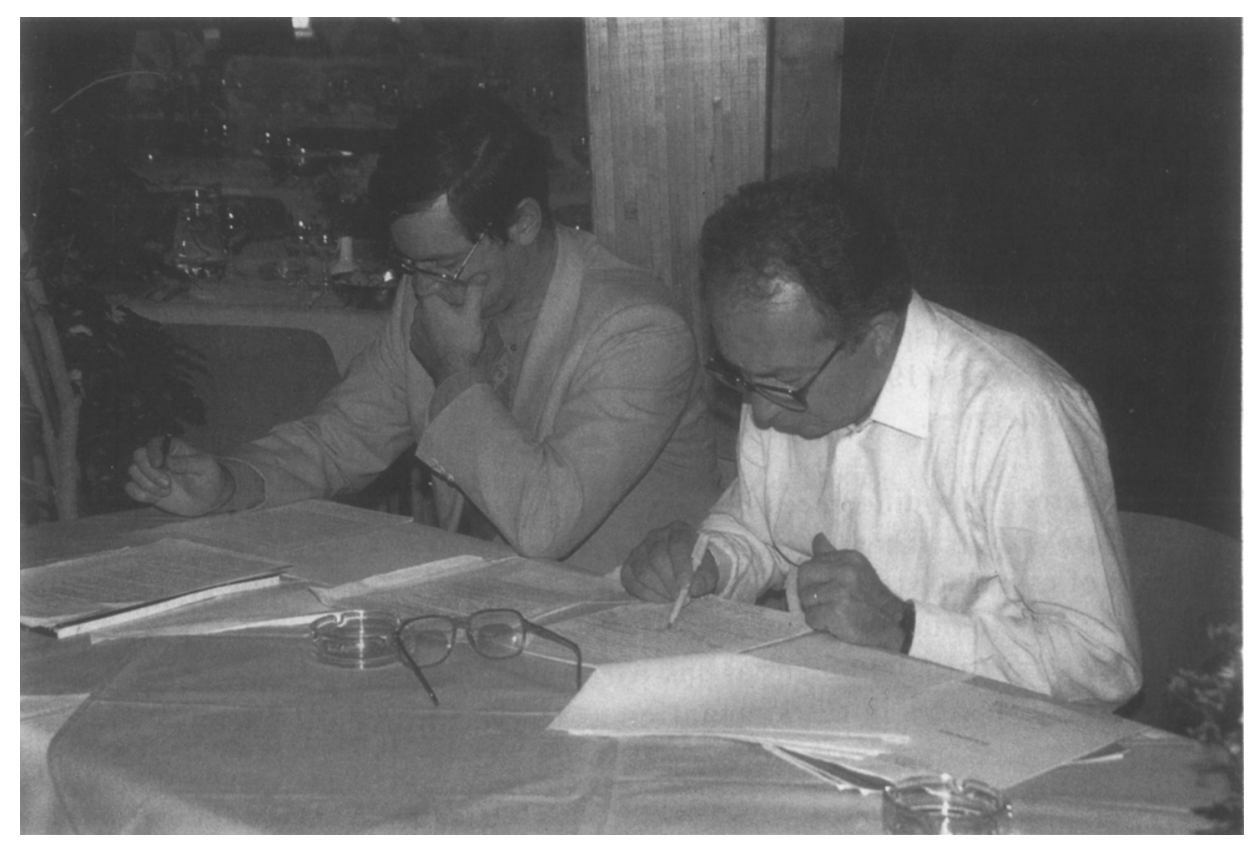

Working at dinner time: W. Zeilinger and F. Bertola 